\title{
Small-intestinal dysfunction accompanies the complex endocrinopathy of human proprotein convertase 1 deficiency
}

\author{
Robert S. Jackson, ${ }^{1}$ John W.M. Creemers, ${ }^{2}$ I. Sadaf Farooqi, ${ }^{3}$ Marie-Laure Raffin-Sanson, ${ }^{4}$ \\ Andrea Varro, ${ }^{5}$ Graham J. Dockray, ${ }^{5}$ Jens J. Holst,${ }^{6}$ Patricia L. Brubaker, ${ }^{7}$ Pierre Corvol, ${ }^{8}$ \\ Kenneth S. Polonsky, ${ }^{9}$ Diane Ostrega, ${ }^{10}$ Kenneth L. Becker, ${ }^{11}$ Xavier Bertagna, ${ }^{4}$ \\ John C. Hutton, ${ }^{12}$ Anne White, ${ }^{13}$ Mehul T. Dattani, ${ }^{14}$ Khalid Hussain, ${ }^{14}$ Stephen J. Middleton, ${ }^{15}$ \\ Thomasina M. Nicole, ${ }^{16}$ Peter J. Milla, ${ }^{14}$ Keith J. Lindley, ${ }^{14}$ and Stephen O’Rahilly ${ }^{3}$
}

${ }^{1}$ Chemical Pathology Department, East Surrey Hospital, Redhill, Surrey, United Kingdom

${ }^{2}$ Department of Human Genetics, University of Leuven and Flanders Interuniversity Institute for Biotechnology, Leuven, Belgium ${ }^{3}$ University of Cambridge Department of Clinical Biochemistry, Addenbrooke's Hospital, Cambridge, United Kingdom ${ }^{4}$ Centre National de la Recherche Scientifique, Institute Cochin de la Genetique Moleculaire, Université René Descartes, Paris, France ${ }^{5}$ Physiological Laboratory, Liverpool University, Liverpool, United Kingdom

${ }^{6}$ Department of Medical Physiology, Panum Institute, Copenhagen, Denmark

${ }^{7}$ Department of Physiology, University of Toronto, Toronto, Ontario, Canada

${ }^{8}$ Institut National de la Santé et de la Recherche Médicale U36, Collège de France, Paris, France

${ }^{9}$ Department of Internal Medicine, Washington University in St. Louis, St. Louis, Missouri, USA

${ }^{10}$ Department of Medicine, University of Chicago, Chicago, Illinois, USA

${ }^{11}$ Department of Medicine, George Washington University, Washington, DC, USA

${ }^{12}$ Barbara Davis Center for Childhood Diabetes, University of Colorado Health Sciences Center, Denver, Colorado, USA

${ }^{13}$ School of Biological Sciences, University of Manchester, Manchester, United Kingdom

${ }^{14}$ Institute of Child Health, London, United Kingdom

${ }^{15}$ Department of Gastroenterology, Addenbrooke's Hospital, Cambridge, United Kingdom

${ }^{16}$ Department of Paediatrics, Yeovil General Hospital, Somerset, United Kingdom

We have previously described the only reported case of human proprotein convertase 1 (PC1) deficiency, in a female (Subject A) with obesity, hypogonadism, hypoadrenalism, and reactive hypoglycemia. We now report the second case of human PC1 deficiency (Subject B), also due to compound heterozygosity for novel missense and nonsense mutations. While both subjects shared the phenotypes of obesity, hypoadrenalism, reactive hypoglycemia, and elevated circulating levels of certain prohormones, the clinical presentation of Subject B was dominated by severe refractory neonatal diarrhea, malabsorptive in type. Subsequent investigation of Subject A revealed marked small-intestinal absorptive dysfunction, which was not previously clinically suspected. We postulate that PC1, presumably in the enteroendocrine cells, is essential for the normal absorptive function of the human small intestine. The differences in the nature and severity of presentation between the two cases cannot readily be explained on the basis of allelic heterogeneity, as the nonsense and missense mutations from both subjects had comparably severe effects on the catalytic activity of PC1. Despite Subject A's negligible PC1 activity, some mature ACTH and glucagonlike peptide $1^{7-36 a m i d e}$ were detectable in her plasma, suggesting that the production of these hormones, at least in humans, does not have an absolute dependence on PC1. The presence of severe obesity and the absence of growth retardation in both subjects contrast markedly with the phenotype of mice lacking PC1 and suggest that the precise physiological repertoire of this enzyme may vary between mammalian species.

J. Clin. Invest. 112:1550-1560 (2003). doi:10.1172/JCI200318784.

Received for publication April 30, 2003, and accepted in revised form September 9, 2003.

Address correspondence to: S. O'Rahilly, Department of Clinical Biochemistry, Hills Road, Addenbrooke's Hospital, Cambridge CB2 2QQ, United Kingdom. Phone: 44-1223-336855; Fax: 44-1223-330160; E-mail: sorahill@hgmp.mrc.ac.uk.

Robert S. Jackson and John W.M. Creemers contributed equally to this work.

Conflict of interest: The authors have declared that no conflict of interest exists.

Nonstandard abbreviations used: proprotein convertase 1 (PC1); proopiomelanocortin (POMC); glucagon-like peptide (GLP); inactive propeptide form of $\mathrm{PC} 1$ (pro-PC1); corticotropin-releasing hormone (CRH); immunoradiometric assay (IRMA); corticotropin-like intermediate lobe peptide (CLIP).

\section{Introduction}

The biosynthesis of many secreted peptides involves limited endoproteolysis of larger, usually inactive, precursors to release the bioactive fragments. A family of serine endoproteases (proprotein convertases) that perform this processing function within the secretory pathway has been defined (1-3). Two members, proprotein convertases 1 and 2 (PC1 and PC2), which show expression confined to the regulated secretory pathway of neuroendocrine tissue, have been particularly closely studied. Although ex vivo experiments indicate that their substrate specificities overlap, in vivo they appear 
to have more distinct functions, evidenced by tissuespecific expression. For example, corticotropes, which express only PC1, cleave proopiomelanocortin (POMC) to adrenocorticotropin (ACTH), while melanotropes, which additionally express PC2, form $\alpha$-melanocyte stimulating hormone ( $\alpha \mathrm{MSH})$ and $\beta$ endorphin (4). Similarly, proglucagon is processed to glucagon-like peptides 1 and 2 (GLP-1 and GLP-2) in intestinal L cells, which express PC1, and to glucagon in pancreatic islet $\alpha$ cells, which express PC2 (see Figure 3a) $(5,6)$.

The functions of mammalian PC1 and PC2 have been further clarified through the generation, by homologous recombination, of mice deficient in one or the other of these enzymes. PC2-null animals have fasting hypoglycemia due to a lack of production of mature glucagon from the pancreatic $\alpha$ cell, and PC1null mice, while sharing some elements of the phenotype reported in humans, uniquely have severely reduced linear growth attributed to failure of growth hormone releasing hormone production (7-9). It is as yet unclear whether these enzymes have an identical range of actions in humans and to what extent dysfunction of these enzymes can contribute to human metabolic and endocrine disease.

We previously reported a woman (Subject A) with marked childhood obesity, hypogonadotropic hypogonadism, postprandial hypoglycemia, hypocortisolemia, and evidence of impaired processing of ACTH and insulin precursors $(9,10)$. She is a compound heterozygote for PC1 mutations: Gly593Arg (GenBank $\mathrm{X} 64810$ ), which causes failure of maturation of the inactive propeptide form of PC1 (pro-PC1) and its retention in the $E R$, and $A \rightarrow C^{+4}$ in the donor splice site of intron 5, resulting in exon skipping and a frameshift and premature stop codon in the catalytic domain. We now describe the second case of congenital PC1 deficiency, in a patient (Subject B) in whom the presenting features were dominated by severe smallintestinal dysfunction. Re-evaluation of Subject A showed that she too had small-intestinal dysfunction, but of a lesser degree, which prompted us to examine whether dissimilarities between the subjects were due to differences in function of the mutant enzymes. Finally, in Subject A, we further evaluated circulating prohormones and products reportedly associated with PC1, to clarify the role of this enzyme in prohormone processing events in humans.

\section{Methods}

Genetic studies in Subject B and construction of vectors expressing mutant PC1

The 14 exons of PC1 and their intronic boundaries in Subject B were bidirectionally sequenced (BigDye Terminator cycle sequencing; Roche Molecular Biochemicals, Mannheim, Germany) using PCR amplified genomic DNA (10). Construction of the Gly593Arg mutant PC1 cDNA has been described previously (10). A FLAG epitope tag (Asp-Tyr-Lys-Asp-Asp-Asp-Asp-Lys) was introduced between the propeptide and the catalytic domain by insertion mutagenesis using Altered Sites II in vitro Mutagenesis Systems (Promega Corp., Madison, Wisconsin, USA). PC1-Ala213del and all other mutations were created using a QuikChange sitedirected mutagenesis kit (Stratagene, La Jolla, California, USA). So that the propeptide could be labeled metabolically with ${ }^{35} \mathrm{~S}$-methionine, Ile86 was replaced by a Met in the co-immunoprecipitation study. WT PC1 and splice-site variant PC1 (PC1-ssv) minigenes were constructed using an approximately $7-\mathrm{kb}$ genomic fragment of PC1 spanning exons 4-6, which was amplified by PCR and cloned in pGEM-T Easy (Promega Corp.). A 4-kb KpnI-KpnI fragment from intron 5 was deleted by digestion before insertion into the above cDNA. This construct and one with the splice-site mutation inserted were cloned into pcDNA3 (Invitrogen Life Technologies, Groningen, The Netherlands) and checked by sequencing.

\section{Cell lines, DNA transfection, and protein analysis}

Medium, serum, and supplements were obtained from Invitrogen Life Technologies, and cells were cultured as previously described (11). CHO-K1 and HEK-293T cells were transfected with FuGENE (Roche Molecular Biochemicals), AtT-20 cells with Lipofectamine (Invitrogen Life Technologies), and $\alpha$ TC1-6, $\beta$ TC 3 , and Neuro2A cells with Lipofectamine 2000 (Invitrogen Life Technologies). Cell labeling, lysis, immunoprecipitation, SDS-PAGE, and Western blotting were performed as previously described (11) using anti-PC1 antibody (Alexis Corp., Läufelfingen, Switzerland) or anti-FLAG M2 (Sigma-Aldrich, Bornem, Belgium).

\section{Assay of PC1 activity}

Recombinant PC1 was immunopurified from cells and medium as previously described (11), except for the lysis buffer (0.5\% Triton X-100, $10 \mu \mathrm{M}$ E-64, $1 \mu \mathrm{M}$ leupeptin, $10 \mu \mathrm{M}$ pepstatin, and $100 \mu \mathrm{M}$ tosyl phenylalanyl chloromethyl ketone; all from Sigma-Aldrich). Recombinant PC1 was selectively detected in cells expressing endogenous PC1 by means of the FLAG epitope tag. To normalize the quantity of enzyme used in activity assays, PC1 expression was determined by overnight labeling of cells to steady-state levels (11) followed by SDS-PAGE analysis of $10 \%$ of the immunoprecipitate with measurement of band intensities using Image Station 440 (Eastman Kodak Co., New Haven, Connecticut, USA). The enzymatic activity of immunoprecipitates was assayed using fluorogenic substrate p-Glu-Arg-Thr-ArgArg-amino methylcoumarin (Peptides International Inc., Louisville, Kentucky, USA) at $37^{\circ} \mathrm{C}(12)$.

\section{Analysis of circulating prohormones} and their products

Proglucagon. Plasma from Subject A was collected during fasting and at 20-minute intervals after the start of a test meal (4MJ: 52\% carbohydrate, $41 \%$ fat, $7 \%$ protein). Proglucagon-derived peptides were measured by RIA 
using antisera specific to free C-terminal glucagon, midsequence glucagon, and amidated C-terminal GLP-1 (5). The results of these assays were consolidated by further RIAs using antisera specific to mid-sequence glucagon (antiserum 4304), free C-terminal glucagon (antiserum 4305), mid-sequence GLP-1 (antiserum 2135), amidated C-terminal GLP-1 (antiserum 89390), and free N-terminal GLP-2 (antiserum 92160) (13). To identify the proglucagon products contributing to the measured immunoreactivities, plasma from Subject A and controls was subjected to size-exclusion gel chromatography, as previously described, prior to RIA of eluted peptides using the above assays $(13,14)$. In addition, eluted $N$-terminal GLP-1 ${ }^{7-36}$ and mid-sequence GLP-2 immunoreactivities were measured by RIA (antisera 93242 and RAS 7167, respectively; Peninsula Laboratories Europe LTD, St. Helens, United Kingdom). GLP-1 forms in plasma were also identified using reverse-phase HPLC with RIA of eluted amidated C-terminal GLP-1 (5). In both systems, peaks were defined by migration relative to standards and by the RIA specificity.

Progastrin. Progastrin and gastrin forms were measured in the plasma collected for proglucagon analysis, by RIA using antisera to C-terminal progastrin uncleaved at Arg94,95 (antiserum L289), amidated Cterminal G17 and G34 gastrins (antiserum L2), intact G17 (antiserum L6), and free N-terminal G17 (antiserum 1295) (15-17). Gastrins were also measured in controls (five male and five female, mean ages 39 and 34 years, respectively) using antisera L289 and L2.

POMC. To study the diurnal and stimulated secretion of POMC, ACTH, and cortisol, we sampled peripheral venous blood throughout a 24-hour period and 20,60, and 120 minutes after i.v. administration of $100 \mu \mathrm{g}$ corticotropin-releasing hormone $(\mathrm{CRH})$. Cortisol was measured by radiocompetitive binding assay using transcortin (18), and ACTH was measured by immunoradiometric assay (IRMA), (ELSA-ACTH; CIS bio international, Gif-sur-Yvette, France). PMOC cross reactivity was such that $7,300 \mathrm{U} / \mathrm{ml}$ gave a signal corresponding to less than $6 \mathrm{pg} / \mathrm{ml}$ ACTH. The plasma POMC IRMA has been described previously (19). One unit of POMC corresponded to $1 \mathrm{pg}$ of POMC protein, and the detection limit was $60 \mathrm{U} / \mathrm{ml}$. Twenty minutes after administration of $\mathrm{CRH}$, corticotropin-like intermediate lobe peptide (CLIP) and ACTH were identified in plasma by extraction on a C18 Sep-Pak cartridge (Waters Associates, Milford, Massachusetts, USA) followed by reversephase HPLC on a $0.46 \mathrm{~cm} \times 25 \mathrm{~cm}$ C8 column (Aquapore RP 300; Brownlee Laboratory, Applied Biosystems, Foster City, California, USA) (20). Fractions were lyophilized and reconstituted in assay buffer prior to ACTH C-terminal RIA, in which CLIP and phosphorylated and nonphosphorylated ACTH showed equal immunoreactivity. Forms of $\beta$ endorphin, $\beta$ lipotropin, and POMC in plasma were identified using size-exclusion gel chromatography prior to $\beta$ endorphin RIA (Peninsula Laboratories Inc., Belmont, California, USA) and POMC IRMA (19). Sephadex G-50 Superfine
(Pharmacia, Amersham Biosciences UK Ltd., Little Chalfont, Buckinghamshire, United Kingdom) in a $0.9 \mathrm{~cm} \times 60 \mathrm{~cm}$ column was equilibrated and developed in $1 \%$ acetic acid with $0.1 \%$ BSA. The column was loaded with $0.8 \mathrm{ml}$ of plasma and eluted at 2.5 $\mathrm{ml} / \mathrm{h}$. Dinitrophenylalanine and ${ }^{125}$ I-labeled IgG were added to the plasma sample as markers of the total and void volumes respectively, in order to calculate fractional elution volume. One-milliliter fractions were lyophilized and reconstituted in assay buffer prior to immunoassay.

Subject B's plasma was examined by direct immunometric assay of cortisol, ACTH, and precursors (POMC and proACTH) in blood collected at 0900 hours (21).

Proinsulin. Insulin forms in Subject B's plasma were assayed by direct immunometric assay, and those in Subject A and her teenage children were analyzed by reverse-phase HPLC with immunoassay of eluted peptides as previously described (9).

Procalcitonin. Serum procalcitonin was assayed in Subject $A$ in triplicate using an immunometric assay (Brahms Diagnostica, Berlin, Germany) with antisera to C-terminal procalcitonin and the centrally located calcitonin sequence (22). This assay cross-reacted with conjoined calcitonin and C-terminal peptide. Procalcitonin was also measured by N-terminal RIA, in which free N-terminal fragment (aminoprocalcitonin) cross-reacts $(22,23)$.

Prorenin. We sampled plasma before the subjects rose in the morning and after they had ambulated for an hour; then we measured active renin in the plasma by IRMA (24). In the same samples, total renin (prorenin plus renin) was determined as the active renin level after trypsinization (25).

\section{Results}

\section{Clinical phenotype of Subject B}

Subject B was a female infant with a normal 46XX karyotype, the first child of non-consanguineous Caucasian parents, who was born after 37 weeks of uncomplicated gestation. Diarrhea started on the third postnatal day and persisted despite oral feeding with a variety of whole protein-, hydrolysate-, and amino acid-based infant formula feeds with differing contents of carbohydrate (including lactose, glucose polymer, glucose, and fructose) and fat (predominantly long-chain triglyceride and medium-chain triglyceride). Diarrhea continued even when she was fed nutritionally inadequate fat-free glucose- and amino acid-based formula feeds. She was found to be hypocortisolemic, but hydrocortisone replacement did not affect the diarrhea. She became grossly obese despite a parenteral calorie intake of less than $50 \%$ of Recommended Daily Allowance. When 18 months old, she suffered a fatal cardiopulmonary arrest of uncertain cause.

Investigations required for clinical management confirmed malabsorption of monosaccharides (both glucose and fructose) and fat. Exocrine pancreatic 
function was preserved (normal fecal elastase). Serial small-intestinal biopsies demonstrated persistent, patchy normoplastic villous atrophy (villus/crypt ratio $1: 1$ to $4: 1$ ), inconsistent with the gross gastrointestinal dysfunction. Some biopsies had a mild increase in lamina propria inflammatory cells, including eosinophils. The surface epithelium was organized normally, with enterocytes and a brush border membrane of normal appearance as observed by routine morphological methods and periodic acid-Schiff and alkaline phosphatase stains. Lactase, sucrase, and trehalase activities were normal in enterocytes of morphologically normal villi. Anti-enterocyte antibodies were absent. In fasting plasma, concentrations of proinsulin $(1,079 \mathrm{pmol} / \mathrm{l}$, normal < 7$)$ and des-64,65 proinsulin (71 pmol/1, normally undetectable) were very high, but levels of insulin $(14 \mathrm{pmol} / \mathrm{l}$, normal $<60)$ and des-31,32 proinsulin $(25 \mathrm{pmol} / \mathrm{l}$, normal $<16)$ were relatively normal. This pattern resembled that reported previously in Subject A (9). Investigation of Subject A's lifelong and frequent episodes of hypoglycemia revealed hypocortisolemia (30-80 $\mathrm{nmol} / \mathrm{l}$, normal $>200$ ) associated with low/normal ACTH (2-3 pmol/1, normal 1-11) and high precursor concentrations (104 pmol/1, normal 5-40). The impaired POMC processing and cleavage of proinsulin between the $\mathrm{B}$ and $\mathrm{C}$ chains, both known activities of PC1 $(3,26)$, suggested that PC1 was defective.

\section{Detection of compound-heterozygous PC1 mutations in Subject B}

Sequencing of PC1 in Subject B and her parents revealed that she was a compound heterozygote for Glu250stop (937G $\rightarrow \mathrm{T}$, GenBank X64810) and Ala213del due to deletion of GCA or CAG (Figure 1a). The former mutation was predicted to truncate the PC1 protein within the catalytic domain, while the latter mutation deletes a highly conserved alanine residue near the catalytically essential His208 (Figure 1b). Subject B's heterozygous parents were clinically normal.

\section{Intestinal phenotype of Subject A}

The association of severe gastrointestinal disturbance with PC1 mutations in Subject B led us to reevaluate the surviving 48-year-old PC1-deficient adult, Subject A. Upon questioning, she revealed a lifelong history of abdominal bloating and alternating diarrhea and constipation. Upper and lower gastrointestinal endoscopy revealed normal appearances, and a duodenal biopsy was morphologically normal. Celiac disease serology was negative. Fasting gastric $\mathrm{pH}$ was normal and responded appropriately to food. Normal fecal elastase levels indicated an absence of pancreatic exocrine insufficiency. However, there was evidence for gross abnormalities of small-intestinal absorptive function, with high-volume diarrhea, steatorrhea, bile salt malabsorption, and impaired vitamin $B_{12}$ uptake even in the presence of administered intrinsic factor (Table 1).

\section{Processing of enteroendocrine cell-derived} prohormones in Subject A

In view of the death of Subject B, investigation of the impact of PC1 deficiency on prohormone processing in enteroendocrine cells focused on Subject A, using established chromatography and immunoassays to examine progastrin, proglucagon, and their products in plasma, during fasting and in response to food.

Progastrin. Progastrin (residues 1-101) is cleaved at Arg57,58 and Arg94,95 to release a peptide that becomes the C-terminally glycine-extended peptide G34gly. Carboxyamidation of G34gly forms G34, some of which is cleaved at Lys74,75 to release the mature heptadecapeptide gastrin G17 (Figure 2a) (15). While fasting plasma progastrin immunoreactivities in Subject A and controls were similar, postprandial concentrations were higher in the subject (Figure $2 \mathrm{~b}$ ). Similarly, carboxyamidated gastrin immunoreactivities were higher postprandially in Subject A than in controls (Figure 2c). To determine whether this arose from increased levels of amidated gastrin intermediates such as G34, G17 was measured with antiserum L6, which reacts preferentially with G17 (16). Though the levels of mature G17 measured with L6 were indeed lower than those of total carboxyamidated gastrin measured with L2, the ratio of L2 to L6 immunoreactivity was within reference limits (2 to 5) (27), suggesting unimpeded cleavage of G34 to G17 at the Lys74,75 site. This view was supported by the finding that plasma free N-terminal G17 immunoreactivity (antiserum 1295) was also within reference limits (data not shown) (16). Thus, despite the apparently normal secretion of mature gastrin, the elevated postprandial progastrin/gastrin ratio suggests some impairment of progastrin processing.

Proglucagon. Plasma free C-terminal glucagon immunoreactivity, which normally reflects glucagon, was $333 \mathrm{pg} / \mathrm{ml} 120$ minutes postprandially, compared with $253 \mathrm{pg} / \mathrm{ml}$ in controls. However, mid-sequence glucagon immunoreactivity was $3,430 \mathrm{pg} / \mathrm{ml}$, compared with $328 \mathrm{pg} / \mathrm{ml}$ in controls, raising the possibility of high plasma levels of proglucagon, glicentin, and/or oxyntomodulin, which also react in this assay (Figure 3a). In view of the reported dependence on PC1 of GLP-1 cleavage from proglucagon $(5,6)$, plasma immunoreactivity of the mature product, amidated C-terminal GLP-1, 60 minutes after food intake was unexpectedly high: $158 \mathrm{pg} / \mathrm{ml}$, compared with $162 \pm 53$ $\mathrm{pg} / \mathrm{ml}$ in controls. These findings were confirmed by the second panel of site-specific RIAs (data not shown). The molecular forms contributing to immunoreactivities were identified by chromatography with RIA of eluted peptides, which showed that the reportedly PC1dependent products GLP-1 1 1-36amide, GLP-17-36amide (the bioactive form), GLP-2, and glicentin were all present in plasma and increased with feeding (Figure 3, b-d). Chromatography peaks consistent with proglucagon (glucagon, GLP-1, and GLP-2 immunoreactivities) and 


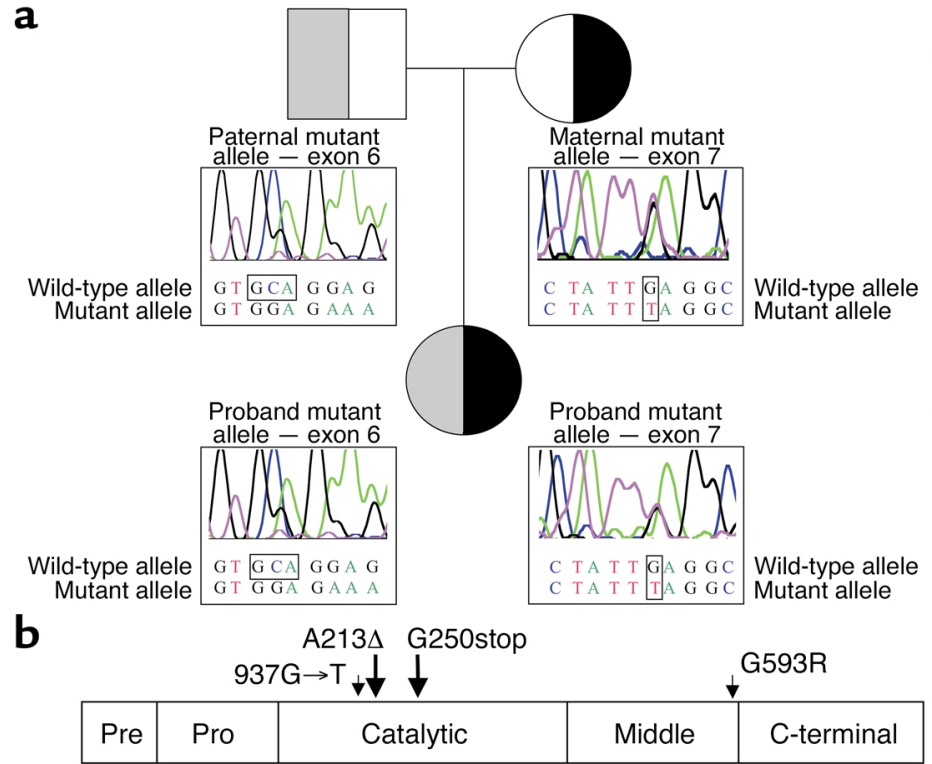

Figure 1

PC1 mutations in Subject B. (a) Bidirectional cycle sequencing of genomic DNA showed Subject B to be compound heterozygous for Ala213del (A213 $\Delta$; deletion of GCA or CAG) and Glu250stop $(937 \mathrm{G} \rightarrow \mathrm{T})$, inherited from her mother and her father, respectively. (b) A linear model of PC1, showing the domains and sites of mutations found in Subjects A and B.

proglucagon minus the C-terminal GLP-2 peptide (glucagon and GLP-1 immunoreactivities only) confirmed observations of high-molecular weight proglucagon forms made with direct plasma assays (Figure 3, c and d, and data not shown). Thus, proglucagon processing was highly impaired despite the continued secretion of mature L cell-type products.

Functional study of mutant PC1s

Expression of recombinant WT PC1, PC1-Gly593Arg, and PC1-Ala213del. WT PC1 is synthesized as inactive proPC1. Cleavage of the propeptide occurs rapidly by an autocatalytic process in the ER, and the carboxyl terminus is cleaved later in the secretory pathway $(28,29)$. In order to study the biosynthesis, maturation, and secretion of mutant PC1 lacking Ala213, the deletion was introduced into PC1 cDNA and overexpressed in CHO-K1 cells, which have been shown to express and secrete active recombinant PC1 $(10,29)$. WT PC1 was detected intracellularly only in its processed form lacking the propeptide, consistent with rapid cleavage (Figure $4 a$, top panels). The majority of immunoreactive PC1, including all carboxy-terminally processed PC1, was secreted. Carboxy-terminal processing was similar to that of recombinant mouse $\mathrm{PC} 1$ expressed in CHODG44 cells (29). Differences in molecular weight and relative amounts are pertinent to human PC1 (data not shown). PC1-Gly593Arg from Subject A was only detected intracellularly as pro-PC1, as reported previously (10). Surprisingly, PC1-Ala213del was detected as both pro-PC1 and PC1, the latter being secreted to some extent. This indicated that propeptide processing of
PC1-Ala213del was not entirely blocked. Similar results were obtained using $\beta$ TC 3 cells, which express endogenous PC1 and efficiently store mature and carboxy-terminally truncated PC1 (Figure 4a, bottom panels). Recombinant PC1 in these cells was selectively detected by means of the FLAG epitope tag.

PC1-Gly593Arg and PC1-Ala213del do not process substrates in trans. Since PC1-Ala213del displayed detectable autoprocessing, studies were performed to test for residual activity for substrates in trans. Neither recombinant mutant PC1 processed detectable amounts of a fluorogenic substrate (Figure $4 \mathrm{~b}$ ). These studies were initially performed using recombinant protein immunopurified from lysates of $\beta$ TC 3 cells, but similar results were obtained using cell lysates and conditioned medium from transfected CHO-K1 cells (data not shown).

Binding and lack of internal cleavage of the propeptide of PC1-Ala213del. Since PC1Ala213del displayed residual activity in cis (autocatalytic propeptide processing) but not in trans, the possibility that the propeptide remained associated with the enzyme after cleavage was investigated. It has been shown that the propeptide of another member of the PC family, furin, remains associated until a second internal autocatalytic cleavage occurs in the more acidic environment of the trans-Golgi network $(30,31)$. This step is necessary for release of the propeptide and hence activation of furin. A similar internal cleavage site is present in the propeptide of PC1 (-P-R-R-S-R-R81 $\downarrow)$. To study the association of the propeptide with PC1, we replaced Ile86 with Met in both PC1 and PC1Ala213del. This substitution did not affect propeptide cleavage (Figure $4 \mathrm{c})$. The intact propeptide $(\sim 10 \mathrm{kDa})$ co-immunoprecipitated with both constructs. However, the internally cleaved form $(\sim 4 \mathrm{kDa})$ was detected only in PC1 and not in PC1-Ala213del. Since only the intact propeptide is a potent inhibitor of PC1 (32), the impaired internal cleavage of the propeptide in PC1Ala213del provides further evidence for the deleterious effect of the mutation. Together, these results show that PC1-Gly593Arg and PC1-Ala213del do not have significant residual activity.

\section{Table 1}

Results of tests of small-intestinal absorptive function in Subject A

\begin{tabular}{lcc}
\hline Test & Result & Reference \\
3-Day fecal weight & $1,516 \mathrm{~g}$ & $<600 \mathrm{~g}$ \\
$\begin{array}{l}\text { Fecal fat content } \\
\text { Seleno-homotaurocholate }\end{array}$ & $59 \mathrm{mmol} / 24 \mathrm{~h}$ & $<18 \mathrm{mmol} / 24 \mathrm{~h}$ \\
$\begin{array}{l}\text { absorption test } \\
\text { Serum } \mathrm{B}_{12}\end{array}$ & $0.8 \%$ & $>8 \%$ \\
$\mathrm{~B}_{12}$ absorption & $201 \mathrm{ng} / \mathrm{l}$ & $>223 \mathrm{ng} / \mathrm{l}$ \\
$\mathrm{B}_{12}$ absorption with intrinsic factor & $7.5 \%$ & $>12 \%$ \\
& $7.5 \%$ & $>12 \%$
\end{tabular}


PC1-ssv is incorrectly spliced. The second allele of Subject $A$ contains a variant at position +4 of the intron 5 donor splice site $(A \rightarrow C)$, which causes skipping of exon 5 from mature mRNA, resulting in a frameshift, 14 ectopic amino acids, and a premature stop codon (10). Although no correctly spliced PC1 was found by RT-PCR, small amounts could have remained undetected. To test this possibility, we constructed WT and splice-site variant (PC1-ssv) minigenes containing introns 4 and 5 . When expressed in CHO-K1 cells, the minigene that contained WT sequence was correctly spliced, resulting in full-length PC1, which was efficiently secreted (Figure 5a). Transfection of PC1-ssv, on the other hand, failed to result in the expression of any detectable full-length PC1. However, the two low-molecular weight (22- and $24-\mathrm{kDa}$ ) proteins that were detectable intracellularly were consistent with exon 5 skipping. The predicted molecular weight of the unglycosylated truncated protein lacking the signal peptide, but containing the propeptide and the ectopic sequences, is $19.574 \mathrm{kDa}$. These immunoreactive bands contain the same core protein but are heterogeneous in Asn-link glycosylation, as deglycosylation by endoglycosidase $\mathrm{F}$ results in a single protein of approximately $19.5 \mathrm{kDa}$ (Figure 5a, right panel). A more sensitive way to detect trace amounts of correctly spliced PC1 is to measure processing activity using a synthetic substrate, but no activity was detected by this method either (Figure 5b). Residual activity was undetectable in conditioned medium and cell lysates from transfected HEK-293T, AtT-20, $\alpha$ TC1-6, $\beta$ TC3, and Neuro2A cells as well (data not shown).

In summary, no processing activity could be detected in the PC1 variants of Subject A or the Ala213del protein of Subject B.

\section{Analysis of further circulating prohormones and products in Subject A}

POMC. The high plasma POMC immunoreactivity and the near-normal ACTH and cortisol immunoreactivities seen previously in Subject A were confirmed and found to be physiologically regulated in terms of diurnal rhythm and response to exogenous $\mathrm{CRH}$, despite the processing abnormality (Figure 6b) (9). The timing of the $\mathrm{CRH}$ response was consistent with secretion via the regulated pathway, and the cortisol response indicated release of bioactive peptide. However, analysis of processing products only in terms of immunoreactivity is potentially misleading, since epitopes may be common to otherwise distinct peptides, e.g., terminally extended forms. Therefore, to identify the POMC products with greater certainty, plasma peptides in Subject A were separated by chromatography prior to site-specific immunoassays. HPLC with RIA of eluted C-terminal ACTH showed that immunoreactive ACTH in plasma was indeed authentic ACTH 1-39 (phosphorylated and nonphosphorylated) (Figure $6 \mathrm{c}$ ). Size-exclusion gel chromatography of plasma with $\beta$ endorphin RIA and POMC IRMA revealed the presence of authentic $\beta$ lipotropin and POMC (Figure 6d). CLIP and $\beta$ endorphin, which are formed by the action of PC2 on POMC (33), were absent, suggesting that PC2 does not substitute for PC1 in processing POMC (Figure 6, $\mathrm{c}$ and d).

Proinsulin. In postprandial plasma, the four healthy heterozygous children of subject $\mathrm{A}$ had ratios of HPLC peaks of proinsulin to des-31,32 proinsulin (respectively PC1 substrate and product after subsequent carboxypeptidase activity) which were 1.65 , $5.25,0.75$, and 1.00. Subject $A$ had a ratio of 2.79 and controls 0.73 in controls 25.7. Thus simple heterozygosity for PC1 mutations did not significantly impair proinsulin processing.

Procalcitonin. Plasma procalcitonin in Subject A was $21 \mathrm{pg} / \mathrm{ml}$ by RIA, compared with $33 \pm 16 \mathrm{pg} / \mathrm{ml}$ in controls, and was less than $300 \mathrm{pg} / \mathrm{ml}$ (normal) by immuno-chemiluminescent assay.
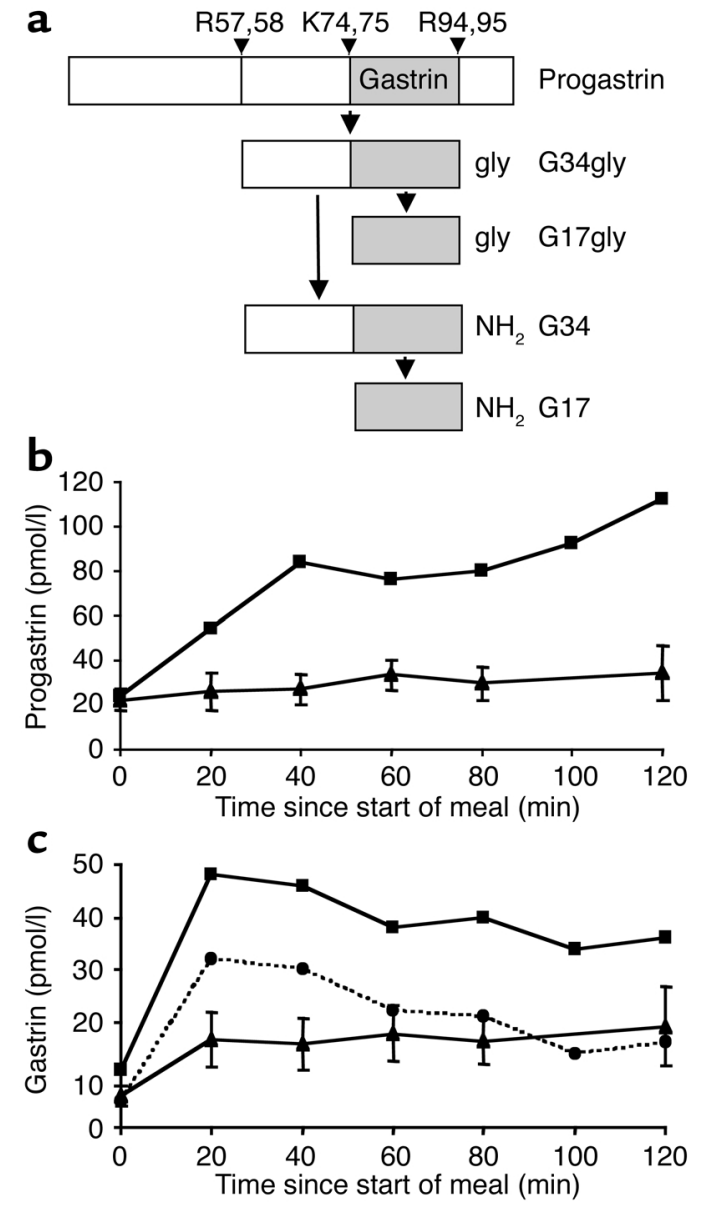

Figure 2

Subject A secreted mature gastrin, but her elevated postprandial plasma progastrin level suggested some impairment of processing. (a) Schematic of sequential cleavage and C-terminal modification of gastrin forms. G17 is mature gastrin. (b and c) Plasma progastrin (b) and total amidated gastrin (c) were measured by RIA using antisera L289 and L2, respectively, in the subject (squares) and ten healthy controls (triangles), before and at 20-minute intervals after the start of a high-fat and -carbohydrate test meal. Mature gastrin, G17, was also measured in the subject, using the highly specific antiserum L6 (circles). Control data are mean $\pm \mathrm{SE}$. 
a

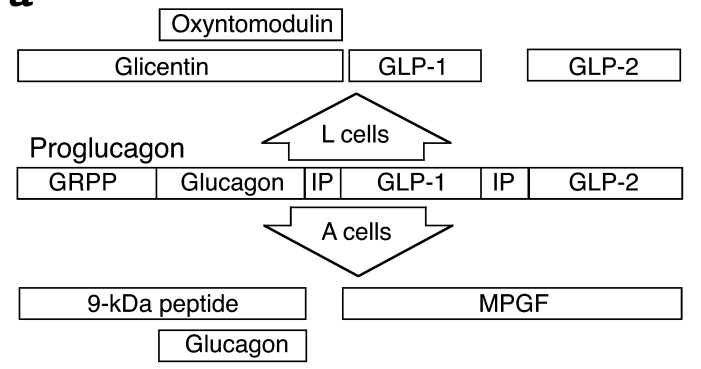

b

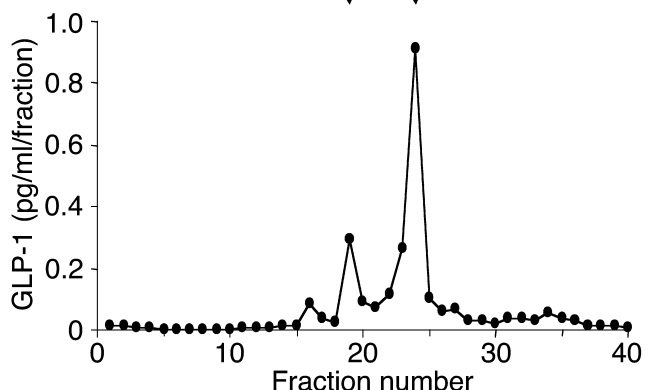

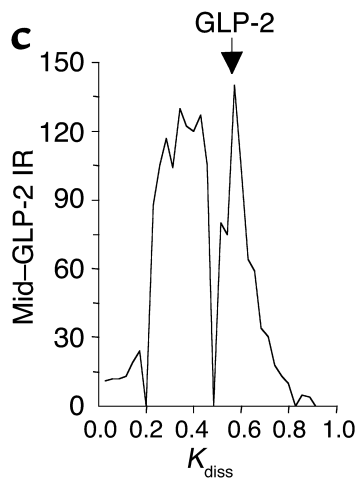

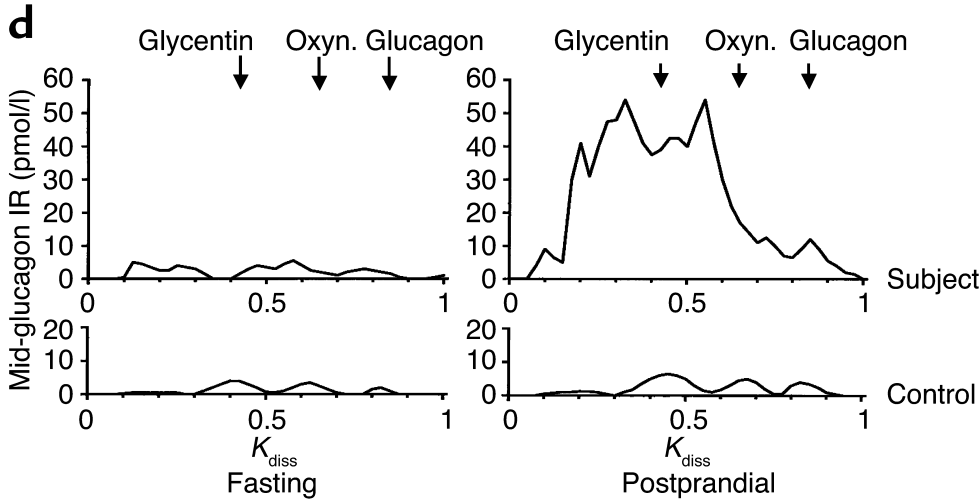

\section{Figure 3}

Subjects A's plasma contained products that normally result from PC1-mediated proglucagon processing. (a) PC1 in intestinal L cells cleaves proglucagon to glicentin, oxyntomodulin, and GLP-1 and -2, while in islet A cells PC2 forms glucagon, 9-kDa peptide, and major proglucagon fragment (MPGF). GRPP, glicentin-related pancreatic peptide; IP, intervening peptide. (b) Reverse-phase HPLC of postprandial plasma with RIA of eluted amidated C-terminal GLP-1 revealed the continued presence of mature GLP-17-36amide. $\downarrow$, GLP-1 standards. (c) GLP-2 also was detectable in postprandial plasma using size-exclusion gel chromatography and RIA of eluted mid-sequence GLP-2 (mid-GLP-2). (d) In response to food, Subject A's apparently normal fasting plasma levels of glicentin, oxyntomodulin, and glucagon rose abnormally high. Sizeexclusion gel chromatography with RIA (antiserum 4304) of eluted mid-sequence glucagon (present in glicentin, oxyntomodulin, and glucagon) was applied to plasma from Subject A (top) and five pooled controls (bottom) during fasting (left) and 1 hour after food intake (right). Oxyn., oxyntomodulin; $K_{\text {diss }}$, coefficient of distribution; $\downarrow$, standards; IR, immunoreactivity.

Prorenin. In Subject A, with ambulation after overnight recumbency, plasma active renin rose from 9 to $16 \mathrm{pg} / \mathrm{ml}$ (vs. a rise from $17 \pm 7$ to $32 \pm 16 \mathrm{pg} / \mathrm{ml}$ in controls), total renin from 138 to $154 \mathrm{pg} / \mathrm{ml}$ (vs. a rise from $206 \pm 93$ to $232 \pm 107 \mathrm{pg} / \mathrm{ml}$ in controls), and active renin as a fraction of the total from $7 \%$ to $10 \%$ (vs. a rise from $9 \% \pm 3 \%$ to $14 \% \pm 6 \%$ in controls). Thus, since total renin is largely prorenin, there was no evidence of impaired processing.

\section{Discussion}

In this article we report the second case of human PC1 deficiency, which confirms that characteristics of this disorder are early-onset severe obesity, impaired POMC and proinsulin processing, hypocortisolemia, and hypoglycemia. The gastroenterological aspects of the illness in Subject B provoked further examination of the first case, in Subject A. The results of those studies lead us to conclude that small-intestinal absorptive dysfunction is a feature of human PC1 deficiency and that this disorder should be considered in the differential diagnosis of intractable neonatal diarrhea.
The evidence for small-intestinal failure in both subjects was strong. Subject B was extensively investigated for known causes of congenital diarrhea; this investigation included multiple small-intestinal biopsies. Fecal analysis revealed failure to absorb multiple dietary sugars and other nutrients. The intestinal failure was so severe and protracted as to require lifelong parenteral feeding. Subject A has had lifelong intermittent diarrhea and constipation, which had been overlooked because of the complexity of her endocrine and metabolic illness. Although the lower small intestine of Subject A was not biopsied, the combination of fat, bile salt, and vitamin $B_{12}$ malabsorption with normal pancreatic exocrine function and gut transit times indicated an absorptive defect in the small intestine.

We hypothesized that PC1 deficiency caused intestinal malfunction through failure of maturation of propeptides within the enteroendocrine cells and nerves that express PC1 throughout the gut (34). Using established chromatographic and immunoassay systems, we studied circulating products of the gut peptides progastrin and proglucagon. The finding of elevated levels of progastrin and proglucagon 

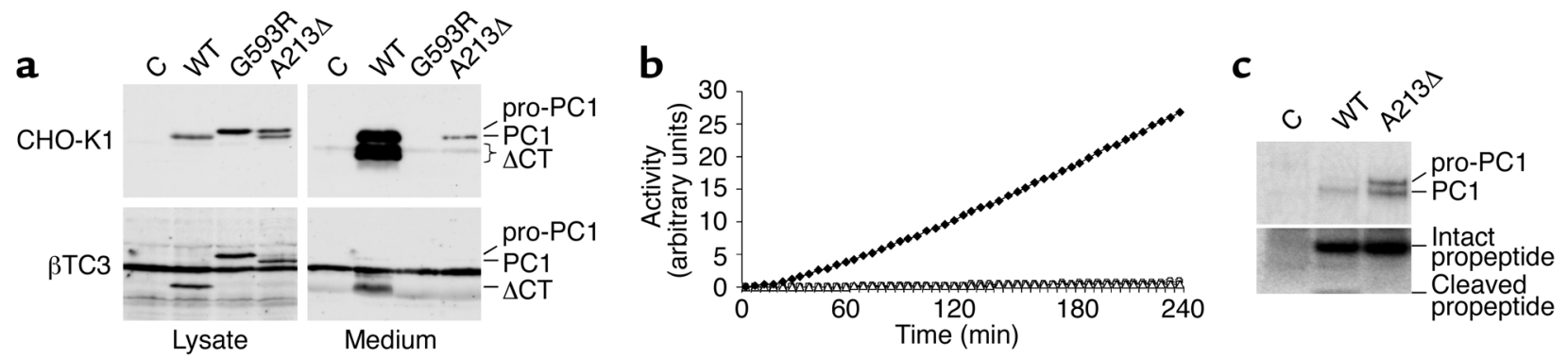

Figure 4

Recombinant PC1-Gly593Arg (PC1-G593R) and PC1-Ala213del (PC1-A213A) were catalytically inactive. (a) Western blot analysis of CHO-K1 and $\beta$ TC3 cells transfected with empty vector (C) or WT PC1, PC1-G593R, or PC1-A213 $\Delta$ cDNA. Recombinant PC1 was detected with an anti-FLAG antibody. $\triangle C T$, C-terminally truncated PC1. (b) Fluorogenic assay of PC1 proteolytic activity using antiFLAG-immunopurified PC1 from lysates of $\beta T C 3$ cells transfected as in a. Open circles, empty vector; filled diamonds, WT PC1 cDNA; open squares, PC1-G593R cDNA; open triangles, PC1-A213 cDNA. Activity was normalized for input of recombinant protein. (c) Coimmunoprecipitation of the propeptide of WT PC1 and PC1-A213 . Radiolabeled cell lysates (30-minute pulse, 1-hour chase) were immunoprecipitated with anti-FLAG antibody as in $\mathbf{b}$. The lower part of the gel was exposed longer to visualize the propeptide.

provided in vivo evidence that, indeed, prohormone processing in enteroendocrine cells was abnormal.

Gastrin is cleaved from progastrin in the neuroendocrine $\mathrm{G}$ cells of the gastric antral and proximal duodenal mucosa. PC1 is a candidate for this role, because its protein and mRNA colocalize with gastrin expression $(15,34)$. Although in Subject A postprandial plasma levels of mature gastrin were within the reference range, progastrin levels were supranormal (Figure 2b), suggesting a role for PC1, but not a critical one. This is consistent with immunocytochemistry in humans, which revealed PC1 to be quantitatively and spatially less distinctly related to gastrin than is PC2 (34).

GLP-2, which is formed in the gut from proglucagon, was an obvious candidate for a role in the gut pathology, because it has trophic effects on small-intestinal epithelium (35). However, direct plasma assays and chromatography/RIA showed that GLP-2 was still present in plasma despite the undetectable activity of PC1 mutants and the reported essential role of PC1 $(3,5)$. Since GLP-1 is formed in amounts equimolar with those of GLP-2, the presence in plasma of mature GLP-17-36amide was also evidence of GLP-2 formation, and vice versa. Although the elevated precursor levels clearly revealed the importance of $\mathrm{PC} 1$ for proglucagon processing, the continued GLP-1 and GLP-2 secretion despite undetectable activity of $\mathrm{PC} 1$ mutants raised the possibility of a redundant PC1-like activity, e.g., PC5, which is widely expressed in the gut (34-38) and might become

\section{Figure 5}

Transfection of a PC1 minigene containing the splice-site variant resulted in undetectable full-sized PC1 protein and catalytic activity. (a) Western blot (left and middle panels) and immunoprecipitation analysis (right panel) of HEK-293T cells transfected with empty vector (C) or PC1 minigene containing either WT or splice-site variant (SSV) sequences. Cells used for immunoprecipitation were pulselabeled for 1 hour. Endo F, endoglycosidase F. (b) Fluorogenic assay of PC1 proteolytic activity using PC1 immunopurified from lysates of $\alpha$ TC1-6 cells transfected as in a. Open circles, empty vector; filled diamonds, PC1 minigene; open squares, PC1 splice-site variant minigene. more active as a result of hyperplasia of $L$ cells analogous to the A cell hyperplasia of PC2 deficiency (39). Interestingly, PC1-null mice too have a gastrointestinal phenotype (bulky moist stools), but GLP-2 was undetectable in gut extracts (8). However, because plasma levels were not measured in the mouse and we could not study the human gut directly, the relationship between the murine and human findings remains unresolved, although it is very likely that, in both species, small-intestinal dysfunction results from disordered prohormone processing within enteroendocrine cells. It is possible that the relative importance of gut neuropeptides and their functions and convertases differs between mice and humans such that the gut dysfunction in humans relates to deficiency of peptides other than GLP-2, e.g., promotilin and pro-PYY products. Plasma levels reflect contributions from multiple sources, so the rise in plasma glucagon after the test meal (Figure 3d) might conceivably have been related to A cells rather than abnormal processing in $L$ cells. Interestingly, serum glucagon was also elevated (2.5fold) in the PC1-null mouse (40).

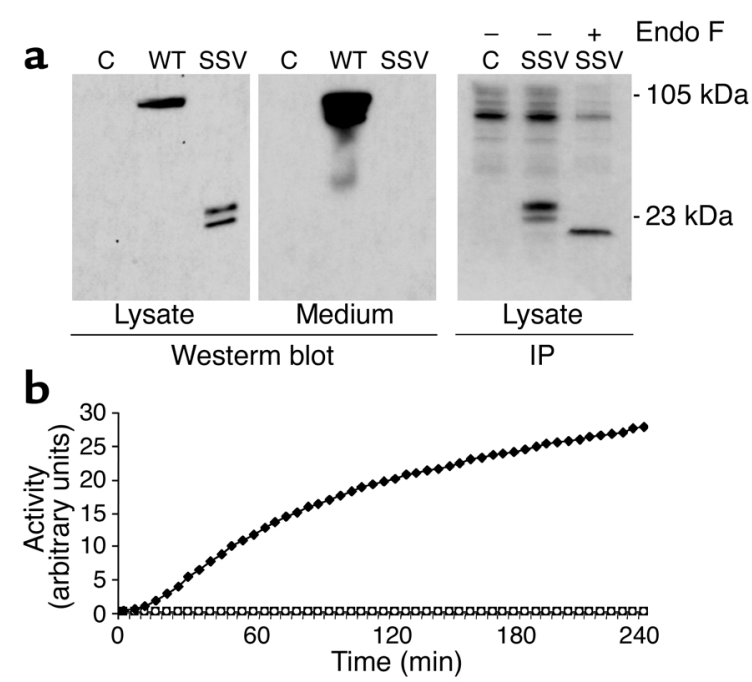


a

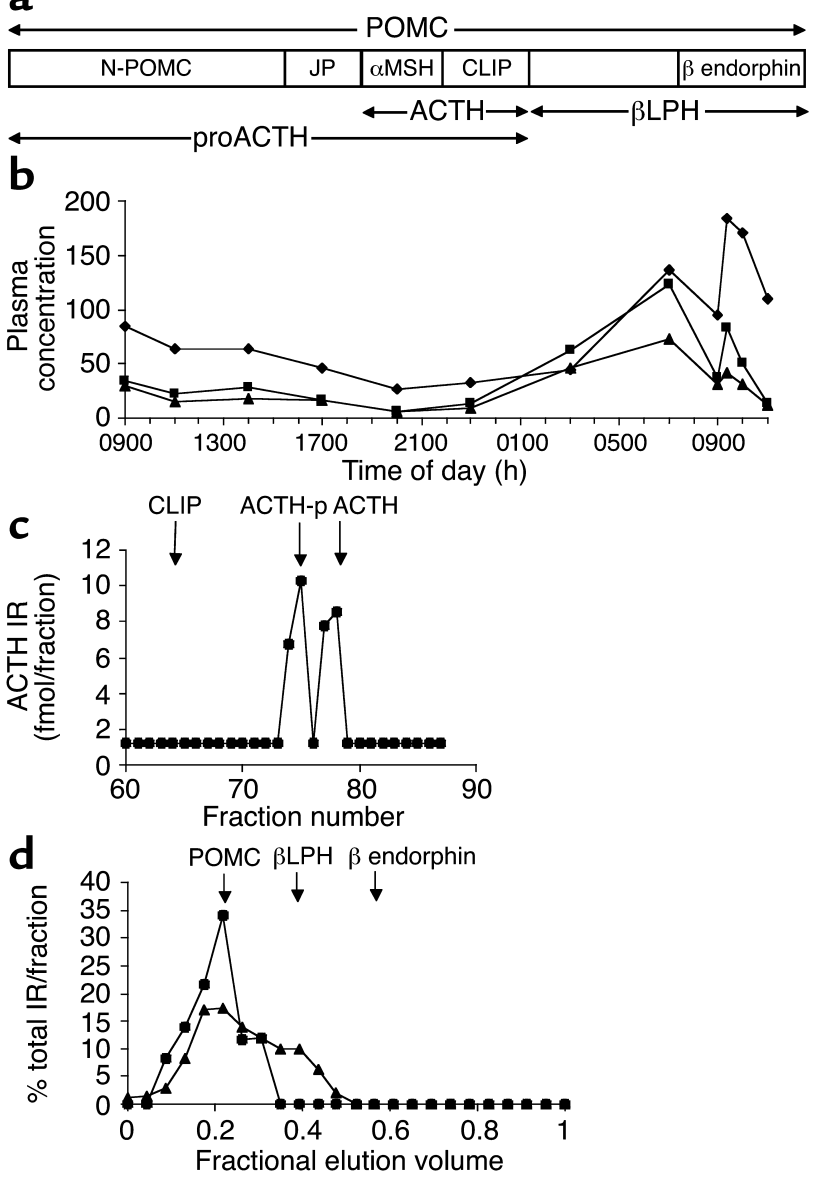

We wondered whether the differences between the two subjects in terms of survival and of severity of gastrointestinal phenotype were explicable on the basis of the degree and type of enzymatic dysfunction produced by their mutations. Results obtained with recombinant PC1-Gly593Arg and PC1-Ala213del strongly suggested that both mutations blocked enzymatic activity toward substrates in trans. PC1Gly593Arg appeared to have lost autoprocessing activity as well. As a consequence, the pro-PC1 was retained in the ER (10), as inactive forms of furin and PC7 have been shown to be (41-43). PC1-Ala213del had reduced autoprocessing activity, but no activity for substrates in trans. Separation of these two activities has also been reported for furin mutants (44) and can be explained in two ways. Firstly, propeptide cleavage is a prerequisite for exit from the ER but does not generate an active enzyme per se. As has been shown for furin, a second internal cleavage is required for release of the inhibitory propeptide. This internal cleavage was found to be impaired in PC1-Ala213del. Secondly, the separation of the activities might be explicable in terms of differences between the kinetics of zero-order autocatalytic cleavage and first-order cleavage of substrates in trans.

Gly593 is conserved in all PCs of all species, and, based on crystal structures of mouse furin and yeast kexin $(45,46)$, it is predicted to be located at the end of

\section{Figure 6}

Physiologically regulated, qualitatively normal POMC products were present in Subject A's plasma. (a) POMC is the precursor to multiple distinct peptides. N-POMC, N-terminal fragment of POMC; JP, joining peptide; $\alpha \mathrm{MSH}, \alpha$-melanocyte stimulating hormone; $\beta \mathrm{LPH}$, $\beta$ lipotropin. (b) Concentrations of plasma cortisol (diamonds; $\mathrm{ng} / \mathrm{ml}$ ), ACTH (squares; pg/ml), and POMC (triangles; $\times 100 \mathrm{U} / \mathrm{ml}$ ) were determined over 24 hours ( 0900 hours to 0900 hours) in Subject A. Immediately after the 0900 -hours sample on day 2 , a $100-\mu \mathrm{g}$ i.v. bolus of CRH was administered. (c) HPLC of plasma sampled 20 minutes after $\mathrm{CRH}$ administration, with RIA of eluted C-terminal $A C T H$, showed peaks of phosphorylated (ACTH-p) and nonphosphorylated ACTH 1-39 (ACTH), confirming the results of direct plasma assays. Neither CLIP (a product of PC2 activity) nor immunoreactive peptides of abnormal size were present, indicating that POMC processing was of normal specificity. $\downarrow$, standards. (d) Size-exclusion gel chromatography of early-morning plasma with $\beta$ endorphin RIA (triangles) and POMC IRMA (circles) of eluted peptides showed $P O M C$ and $\beta$ lipotropin to be present but not $\beta$ endorphin, which is a product of processing of POMC by PC2. Results are expressed as percentages of the total immunoreactivity eluted. $\downarrow$, standards.

a $\beta$-strand. Because of its tight fit, replacement with a bulky arginine is likely to disturb existing salt bridges and cause deformation of the $\beta$-sheet. Mutagenesis studies have recently indicated that even substitution by a small amino acid like alanine is not tolerated (47). Ala213 is predicted to be located within the same hydrophobic helix as the catalytic His 208 (45, 46, 48, 49). Deletion of Ala213 shortens the helix, which will be pulled into the molecule. The charged Glu215 at one end of the helix may prevent movement there, forcing dislocation of His 208 on the other end. This might be expected to reduce catalytic activity and destabilize the protein through distortion of the local structure. Indeed, both PC1-Gly593Arg and PC1-Ala323del, but not WT PC1, were seen in immunoprecipitations (data not shown) to bind BiP, an ER chaperone that binds to unfolded and misfolded proteins and prevents their export from the ER (50).

The splice-site mutation in intron 5 of Subject $A$ was previously shown by RT-PCR to cause exon skipping of exon 5 (10). Here we have shown by a more robust method in multiple cell types that no correctly spliced mRNA, and hence no active PC1, was produced. Only a truncated protein, the predicted translation product of mRNA devoid of exon 5, was seen. This protein and the truncated protein encoded by the Glu250stop mutant allele in Subject B both lack the serine residue known to be essential for serine proteases and will therefore not be catalytically active. In conclusion, these in vitro data provide strong evidence for the complete absence of residual PC1 activity in both patients, and, therefore, differences in clinical presentation are likely to reflect the influence of genetic and environmental background.

The recent description of mice rendered null for PC1 by homologous recombination confirmed features of PC1 deficiency first reported in humans, such as defective proinsulin and POMC processing $(9,40)$. However, 
unlike humans, who showed severe early-onset obesity and normal linear growth, PC1-null mice are not obese but are growth retarded (8). This has been attributed to impaired growth hormone releasing hormone processing that results in low circulating growth hormone levels. In contrast, serum IGF1 levels in both subjects were in the healthy reference range (data not shown), indicating that exposure of the liver to circulating growth hormone in these humans is relatively normal. The absence of obesity in the $\mathrm{PC} 1 \mathrm{KO}$ mouse is surprising given the severe abnormalities of $\mathrm{POMC}$ processing and the importance of POMC-derived peptides in the control of energy balance, evidenced by the obesity of POMC-null mice (51). The relative importance of PC1 for peptides signaling the anabolic and catabolic aspects of energy homeostasis may differ between humans and mice, such that loss of PC1 function in humans has a more adverse effect on weight-loss signals.

Can we gain any further insights into the nature of the processing events that occur in vivo in humans from the study of these "experiments of nature"? The availability of relatively large volumes of plasma from humans allows detailed analysis of circulating molecular species that may be more problematic in mice. Despite the apparently complete absence of functional PC1 in both subjects, they both secreted bioactive ACTH. Detailed study of Subject A showed that it was indeed authentic ACTH and not an immunoreactive but aberrant form resulting from cleavage of POMC at abnormal sites. Interestingly, the PC1-null mouse did not confirm the continued secretion of ACTH seen in PC1-deficient humans $(8,9)$. Although murine circulating corticosterone levels were normal, consistent with secretion of ACTH and human observations, ACTH was reportedly absent from murine pituitary extracts and pulse-labeled pituitaries. Since murine circulating ACTH was not measured, the source of the ACTH bioactivity and the apparent species difference remains unclear. What might substitute for $\mathrm{PC} 1$ in processing POMC to ACTH within corticotropes in humans, albeit with reduced efficiency? It is unlikely that PC2 was compensating, as none of the classic products of PC2-mediated cleavage were detectable in the circulation. There are additional processing activities in corticotropes that might compensate, e.g., POMC-converting enzyme (52, 53), PC5A, furin, and PACE4 (36-38); however, not all of these are targeted to stored neurosecretory granules (54) or appear to have relevant activity (55).

Although PC1 efficiently processes prorenin in experimental systems (56), the plasma prorenin/active renin ratio in Subject A was normal, suggesting that PC1 is redundant for prorenin processing in vivo. Indeed, this may be a function of PC5 $(57,58)$. There was also no evidence of impaired processing of procalcitonin despite the reported strong PC1 immunostaining (34) of thyroid parafollicular $\mathrm{C}$ cells that secrete calcitonin.

Ratios of plasma proinsulin to des-31,32 proinsulin (effectively PC1 substrate and product, respectively) in Subject A's children, who were heterozygous for PC1 mutations, were the same as in controls, suggesting that heterozygous PC1 defects do not significantly impede insulin biosynthesis in humans. In contrast, pulse-chase analysis of proinsulin processing in islets of heterozygous PC1-null mice showed increased proinsulin II levels, consistent with impaired processing (37). Clarification of this apparent species difference in the impact of heterozygosity requires similar metabolic-labeling studies to be conducted on human islets, but the tissue is inaccessible. Differences in cleavage sites between human proinsulin and mouse proinsulin II at the P4 position may explain this discrepancy.

In summary, we describe the second case of human PC1 deficiency, which has helped refine the phenotype of this rare but illuminating genetic disorder. The gastroenterological findings in both subjects demonstrate, for the first time to our knowledge, that normal human intestinal absorptive function is dependent on PC1 activity. The persistent production of hormones such as ACTH and GLP-1 in the absence of any functional PC1 suggests that other enzymes can partially compensate for lack of this convertase in humans.

\section{Acknowledgments}

We thank the Association of Clinical Biochemists (R.S. Jackson), Fonds voor Wetenschappelijk OnderzoekVlaanderen (J.W.M. Creemers), the Wellcome Trust (S. O'Rahilly and I.S. Farooqi), Ipsen Biotech (M.-L. Raffin-Sanson), the United Kingdom Medical Research Council (A. Varro and G.J. Dockray), the NIH (grant P30 DK57516; J.C. Hutton), and the Canada Institutes of Health Research (P.L. Brubaker) for grant support. We thank F. Girard, Richard H. Snider, S. Meulemans, and R. Siezen for their advice and assistance.

\footnotetext{
1. Wilson, H.E., and White, A. 1998. Prohormones: their clinical relevance. Trends Endocrinol. Metab. 9:396-402.

2. Steiner, D. 1998. The proprotein convertases. Curr. Opin. Chem. Biol. 2:31-39.

3. Rouille, Y., et al. 1995. Proteolytic processing mechanisms in the biosynthesis of neuroendocrine peptides: the subtilisin-like proprotein convertases. Front. Neuroendocrinol. 16:322-361.

4. Bertagna, X. 1994. Proopiomelanocortin-derived peptides. Endocrinol. Metab. Clin. North Am. 23:467-485.

5. Dhanvantari, S., Seidah, N.G., and Brubaker, P.L. 1996. Role of prohormone convertases in the tissue-specific processing of proglucagon. $\mathrm{Mol}$. Endocrinol. 10:342-355.

6. Rouille, Y., Kantengwa, S., Irminger, J.C., and Halban, P.A. 1997. Role of the prohormone convertase PC 3 in the processing of proglucagon to glucagon-like peptide 1. J. Biol. Chem. 272:32810-32816.

7. Furuta, M., et al. 1997. Defective prohormone processing and altered pancreatic islet morphology in mice lacking active PC2. Proc. Natl. Acad. Sci.U. S. A. 94:6646-6651.

8. Zhu, X., et al. 2002. Disruption of PC1/3 expression in mice causes dwarfism and multiple neuroendocrine peptide processing defects. Proc. Natl. Acad. Sci. U. S. A. 99:10293-10298.

9. O'Rahilly, S., et al. 1995. Impaired processing of prohormones associated with abnormalities of glucose homeostasis and adrenal function. N. Engl. J. Med. 23:1386-1390.

10. Jackson, R.S., et al. 1997. Obesity and impaired prohormone processing associated with mutations in the human prohormone convertase 1 gene. Nat. Genet. 16:303-306.

11. Creemers, J.W., van de Loo, J.W., Plets, E., Hendershot, L.M., and Van De Ven, W.J. 2000. Binding of BiP to the processing enzyme lymphoma proprotein convertase prevents aggregation, but slows down maturation. J. Biol. Chem. 275:38842-38847.

12. Creemers, J.W.M., Bailyes, E.M., Lindberg, I., and Hutton, J.C. 1999.
} 
Proteolytic processing. In Post-translational processing: a practical approach. B.D. Hames and S.J. Higgins, editors. Oxford University Press. Oxford, United Kingdom. 205-223.

13. Damholt, A.B., Buchan, A.M., Holst, J.J., and Kofod, H. 1999. Proglucagon processing profile in canine $\mathrm{L}$ cells expressing endogenous prohormone convertase $1 / 3$ and prohormone convertase 2. Endocrinology. 140:4800-4808

14. Holst, J.J., et al. 1994. Proglucagon processing in porcine and human pancreas. J. Biol. Chem. 269:18827-18833.

15. Varro, A., et al. 1997. Gastrin biosynthesis in the antrum of patients with pernicious anaemia. Gastroenterology. 112:733-741.

16. Dockray, G.J., and Taylor, I.L. 1976. Heptadecapeptide gastrin: measurement in blood by specific radioimmunoassay. Gastroenterology. 71:971-977.

17. Dockray, G.J., and Walsh, J.H. 1975. Amino terminal gastrin fragment in serum of Zollinger-Ellison syndrome patients. Gastroenterology. 68:222-230.

18. Laudat, M.H., et al. 1987. The hormonal state of pregnancy: modification of cortisol and testosterone. Ann. Endocrinol. (Paris). 48:334-338.

19. Raffin-Sanson, M.L., et al. 1996. High plasma proopiomelanocortin in aggressive adrenocorticotropin-secreting tumors. J. Clin. Endocrinol. Metab. 81:4272-4277.

20. Massias, J.F., Hardouin, S., Vieau, D., Lenne, F., and Bertagna, X. 1994. Phosphorylated forms of adrenocorticotropin and corticotropin-like intermediary lobe peptide in human tumors. Eur. J. Endocrinol. 131:341-346.

21. Crosby, S.R., Stewart, M.F., Ratcliffe, J.G., and White, A. 1988. Direct measurement of the precursors of adrenocorticotropin in human plasma by two-site immunoradiometric assay. J. Clin. Endocrinol. 67:1272-1277.

22. Whang, K.T., et al. 1998. Serum calcitonin precursors in sepsis and systemic inflammation. J. Clin. Endocrinol. Metab. 83:3296-3301.

23. Snider, R.H., Nylen, E.S., and Becker, K.L. 1997. Procalcitonin and its component peptides in systemic inflammation: immunochemical characterization. J. Investig. Med. 45:552-560.

24. Simon, D., et al. 1987. Measurement of active renin by the $4 \mathrm{G} 1$ antihuman renin monoclonal antibody. Clin. Exp. Hypertens. A. 9:1333-1340.

25. Sealey, J.E., Atlas, S.A., Laragh, J.H., Oza, N.B., and Ryan, J.W. 1979. Activation of a prorenin-like substance in human plasma by trypsin and by urinary kallikrein. Hypertension. 1:179-189.

26. Hutton, J.C. 1994. Insulin secretory granule biogenesis and the proinsulin-processing endopeptidases. Diabetologia. 37(Suppl. 2):S48-S56.

27. Nemeth, J., Varro, A., Bridson, J., Walker, R., and Dockray, G.J. 1992. Increased tissue concentrations of the gastrin precursor in patients treated with omeprazole. Eur. J. Clin. Invest. 22:638-644.

28. Zhou, Y., and Lindberg, I. 1994. Enzymatic properties of carboxyl-terminally truncated prohormone convertase 1 (PC1/SPC3) and evidence for autocatalytic conversion. J. Biol. Chem. 269:18408-18413.

29. Zhou, Y., and Lindberg, I. 1993. Purification and characterization of the prohormone convertase PC1(PC3). J. Biol. Chem. 268:5615-5623.

30. Anderson, E.D., VanSlyke, J.K., Thulin, C.D., Jean, F., and Thomas, G. 1997. Activation of the furin endoprotease is a multiple-step process: requirements for acidification and internal propeptide cleavage. $E M B O J$. 16:1508-1518.

31. Anderson, E.D., et al. 2002. The ordered and compartment-specific autoproteolytic removal of the furin intramolecular chaperone is required for enzyme activation. J. Biol. Chem. 277:12879-12890.

32. Boudreault, A., Gauthier, D., and Lazure, C. 1998. Proprotein convertase PC1/3-related peptides are potent slow tight-binding inhibitors of murine PC1/3 and Hfurin. J. Biol. Chem. 273:31574-31580.

33. Zhou, A., Bloomquist, B.T., and Mains, R.E. 1993. The prohormone convertases PC1 and PC2 mediate distinct endoproteolytic cleavages in a strict temporal order during proopiomelanocortin biosynthetic processing. J. Biol. Chem. 268:1763-1769.

34. Scopsi, L., Gullo, M., Rilke, F., Martin, S., and Steiner, D.F. 1995. Proprotein convertases (PC1/PC3 and PC2) in normal and neoplastic human tissues: their use as markers of neuroendocrine differentiation. J. Clin. Endocrinol. Metab. 80:294-301.

35. Drucker, D.J. 1998. Glucagon-like peptides. Diabetes. 47:159-169.

36. Lusson, J., et al. 1993. cDNA structure of the mouse and rat subtilisin/kexin-like PC5: a candidate proprotein convertase expressed in endocrine and non-endocrine cells. Proc. Natl. Acad. Sci. U. S. A. 90:6691-6695.

37. Dong, W., et al. 1995. Distinct mRNA expression of the highly homologous convertases PC5 and PACE4 in the rat brain and pituitary. J. Neurosci. 15:1778-1796.

38. Seidah, N.G., Chretien, M., and Day, R. 1994. The family of subtilisin/kexin like pro-protein and pro-hormone convertases: divergent or shared functions. Biochimie. 76:197-209.

39. Furuta, M., et al. 1997. Defective prohormone processing and altered pancreatic islet morphology in mice lacking active SPC2. Proc. Natl. Acad. Sci. U. S. A. 94:6646-6651.

40. Zhu, X., et al. 2002. Severe block in processing of proinsulin to insulin accompanied by elevation of des-64,65 proinsulin intermediates in islets of mice lacking prohormone convertase 1/3. Proc. Natl. Acad. Sci. U. S. A. 99:10299-10304.

41. Molloy, S.S., Thomas, L., Van Slyke, J.K., Sternberg, P.E., and Thomas, G. 1994. Intracellular trafficking and activation of the furin proprotein convertase: localization to the TGN and recycling from the cell surface. EMBO J. 13:18-33.

42. Creemers, J.W., et al. 1995. Endoproteolytic cleavage of its propeptide is a prerequisite for efficient transport of furin out of the endoplasmic reticulum. J. Biol. Chem. 270:2695-2702.

43. van de Loo, J.W., et al. 1997. Biosynthesis, distinct post-translational modifications, and functional characterization of lymphoma proprotein convertase. J. Biol. Chem. 272:27116-27123.

44. Creemers, J.W., et al. 1993. Modulation of furin-mediated proprotein processing activity by site-directed mutagenesis. J. Biol. Chem. 268:21826-21834.

45. Henrich, S., et al. 2003. The crystal structure of the proprotein processing proteinase furin explains its stringent specificity. Nat. Struct. Biol. 10:520-526.

46. Holyoak, T., et al. 2003. 2.4 A resolution crystal structure of the prototypical hormone-processing protease Kex 2 in complex with an Ala-LysArg boronic acid inhibitor. Biochemistry. 42:6709-6718.

47. Ueda, K., et al. 2003. Mutational analysis of predicted interactions between the catalytic and P domains of prohormone convertase 3 (PC3/PC1). Proc. Natl. Acad. Sci. U. S. A. 100:5622-5627.

48. Siezen, R.J., Creemers, J.W., and Van de Ven, W.J. 1994. Homology modelling of the catalytic domain of human furin. A model for the eukaryotic subtilisin-like proprotein convertases. Eur. J. Biochem. 222:255-266.

49. Lipkind, G., Gong, Q., and Steiner, D.F. 1995. Molecular modeling of the substrate specificity of prohormone convertases SPC2 and SPC3. J. Biol. Chem. 270:13277-13284.

50. Fewell, S.W., Travers, K.J., Weissman, J.S., and Brodsky, J.L. 2001. The action of molecular chaperones in the early secretory pathway. Annu. Rev. Genet. 35:149-191.

51. Yaswen, L., Diehl, N., Brennan, M.B., and Hochgeschwender, U. 1999. Obesity in the mouse model of pro-opiomelanocortin deficiency responds to peripheral melanocortin. Nat. Med. 5:984-985.

52. Loh, Y.P., Parrish, D.C., and Tuteja, R. 1985. Purification and characterization of a paired basic residue-specific pro-opiomelanocortin converting enzyme from bovine pituitary intermediate lobe secretory vesicles. J. Biol. Chem. 260:7194-7205.

53. Loh, Y.P., and Cawley, N.X. 1995. Processing enzymes of pepsin family: yeast aspartic protease 3 and pro-opiomelanocortin converting enzyme. Methods Enzymol. 248:136-146.

54. Mains, R.E., et al. 1997. PACE4: a subtilisin-like endoprotease with unique properties. Biochem. J. 321:587-593.

55. Seidah, N.G., et al. 1999. The subtilisin/kexin family of precursor convertases. Emphasis on PC1, PC2/7B2, POMC and the novel enzyme SKI-1. Ann. N. Y. Acad. Sci. 885:57-74.

56. Jutras, I., Seidah, N.G., Reudelhuber, T.L., and Brechler, V. 1997. Two activation states of the prohormone convertase PC1 in the secretory pathway. J. Biol. Chem. 272:15184-15188.

57. Mercure, C., Jutras, I., Day, R., Seidah, N.G., and Reudelhuber, T.L. 1996. Prohormone convertase PC5 is a candidate processing enzyme for prorenin in the human adrenal cortex. Hypertension. 28:840-846.

58. Nakagawa, T., et al. 1993. Identification and functional expression of a new member of the mammalian kex-2 like processing endoprotease family: its striking structural similarity to PACE 4. J. Biochem. (Tokyo). 113:132-135. 